Standing Wave Linear Accelerators:

An Investigation of the Fundamental Field Stability and Tuning Characteristics

\title{
Ellie Lin
}

Office of Science, Energy Research Undergraduate Laboratory Fellowship

University of Texas at Austin

Stanford Linear Accelerator Center

Menlo Park, California

August 13, 2002

Prepared in partial fulfillment of the requirements of the Office of Science, Department of Energy Energy Research Undergraduate Laboratory Fellowship under the direction of

R.M. Jones in Accelerator Research Department - A at Stanford Linear Accelerator Center. 


\section{Table of Contents}

1.0 Introduction 1

2.0 Materials and Methods 3

2.1 Tuning of Modes for the Standing Wave Structure 3

2.2 Wakefields in Standing Wave and Traveling Wave Structures 6

$\begin{array}{ll}3.0 \text { Results } & 7\end{array}$

3.1 Tuning of Modes for the Standing Wave Structure $\quad 7$

3.2 Wakefields in Standing Wave and Traveling Wave Structures $\quad 11$

$\begin{array}{ll}\text { 4.0 Discussion and Conclusions } & 13\end{array}$

4.1 Tuning of Modes for the Standing Wave Structure 13

4.2 Wakefields in Standing Wave and Traveling Wave Structures 15

$\begin{array}{ll}5.0 \text { Acknowledgements } & 16\end{array}$

$\begin{array}{ll}\text { 6.0 Appendix A } & 16\end{array}$

$\begin{array}{ll}7.0 \text { Appendix B } & 19\end{array}$

$\begin{array}{ll}\text { 8.0 Literature Cited } & 22\end{array}$ 


\subsection{Introduction}

The first accelerators were designed as a tool in high-energy particle physics. Their development has given rise to numerous applications in industry, such as materials processing, sterilization, food preservation, and radiopharmaceutical product generation (Barbalat, 1994). Modern day linear accelerators for particle physics accelerate multiple bunches of electrons and positrons up to $50 \mathrm{GeV}$. Accelerators of the next generation, such as the Next Linear Collider (NLC), aim to accelerate the bunches initially to a center of mass of $500 \mathrm{GeV}$ and later to $1.5 \mathrm{TeV}$ (Decking 2001, Miyamoto 2002, Phinney 2002). The NLC will operate under gradient fields on the order of $70 \mathrm{MV} / \mathrm{m}$ (Phinney, 2002). For all accelerators, two issues are fundamental for their construction: maximizing the efficiency of acceleration while, at the same time, preserving the luminosity of the beam. These issues are critically important in the design of the NLC.

A linear accelerator operates as follows: An electron gun fires electrons into a structure that bunches the electrons and tightly focuses the beam. At the same time, a radiofrequency wave is fed into the accelerating structure. The electron bunches enter the accelerating structure in phase with the crest of the radiofrequency wave in order to achieve maximum energy.

There are two principal types of accelerating structures: traveling wave (TW) and standing wave (SW). The electromagnetic wave in a TW structure travels in one direction; the electromagnetic wave in a SW structure travels in two directions. Many TW structures have been designed for the NLC, but recent experiments indicate that TW structures suffer from electrical breakdown at high gradients (Miller et. al., 2001). To address this problem, SW structures are being considered as the alternative for the NLC (Jones and Miller et. al., 2002). The input power 
required for an accelerating cavity increases with the length of the cavity (Miller et. al., 2001). Since SW structures can be made arbitrarily short without sacrificing efficiency, SW structures require a lower gradient to achieve the same accelerations as their TW wave counterparts.

Luminosity is the reaction rate per unit cross section. Luminosity is defined by the following equation, Luminosity $=\frac{\mathrm{fN}_{1} \mathrm{~N}_{2}}{\sqrt{\beta_{\mathrm{x}} \beta_{\mathrm{y}} \varepsilon_{\mathrm{x}} \varepsilon_{\mathrm{y}}}}$, where $\mathrm{f}$ is the collision frequency, $\mathrm{N}_{1}$ and $\mathrm{N}_{2}$ are the number of target particles, $\beta$ is the focusing function of the linac, and $\varepsilon$ is the emittance. The smaller and more concentrated the beam, the greater the number of collisions that will occur. Focusing, mainly by quadrupole magnets, helps to maintain high luminosity. However, longrange transverse wakefields can decrease the beam's luminosity and can lead to a beam break up (BBU) instability (Yokoya, 1986).

Wakefields are electromagnetic fields left by leading particles that can interact with subsequent particles. An off-axis bunch can excite a mode that deflects following bunches (Henke, 1994). At high gradients, the traverse component of wakefields given off by deflected bunches can cause bunches of electrons to oscillate with increasing amplitudes, possibly leading to severe damage to the accelerator (Jones and Baboi et al., 2002). Wakefields can be separated into different multipoles. The multipole wakefield that contributes the most to BBU is the dipole wakefield (Jones and Baboi et al., 2002). It is important to understand the behavior of wakefields in order to properly damp them.

Many different studies are being done to ensure the optimal performance of the NLC. This project involved studying the two issues essential to the design of a linear accelerator: 
maximizing the efficiency of acceleration while, at the same time, preserving the luminosity of the beam. SW accelerator cavities were modeled with equivalent circuits and investigated in the $\pi$ and $\pi / 2$ modes of operation. Several bands of the dipole wakefield were then studied in both the SW and TW structures.

\subsection{Materials and Methods}

\subsection{Tuning of Modes for the Standing Wave Structure}

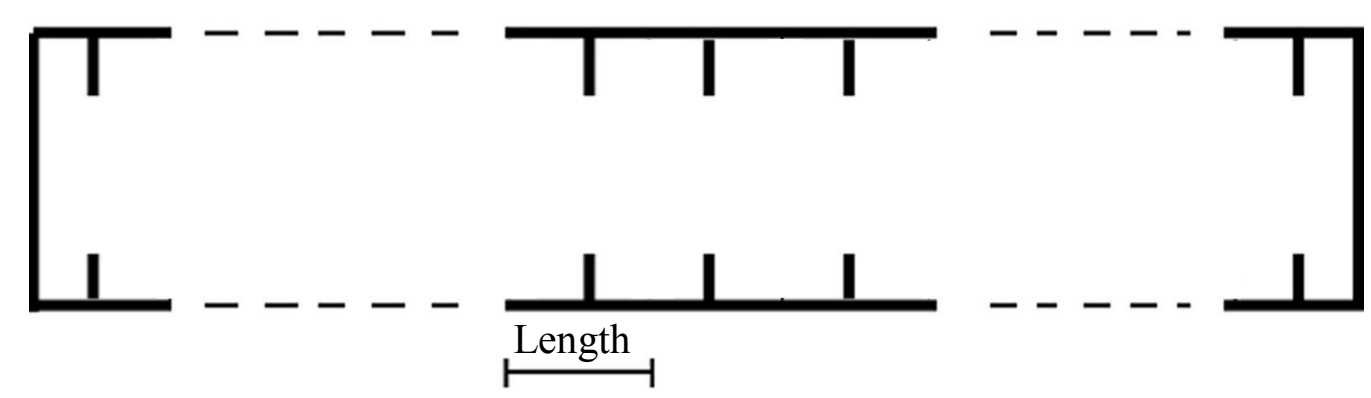

Figure 1: Schematic of standing wave accelerator structure

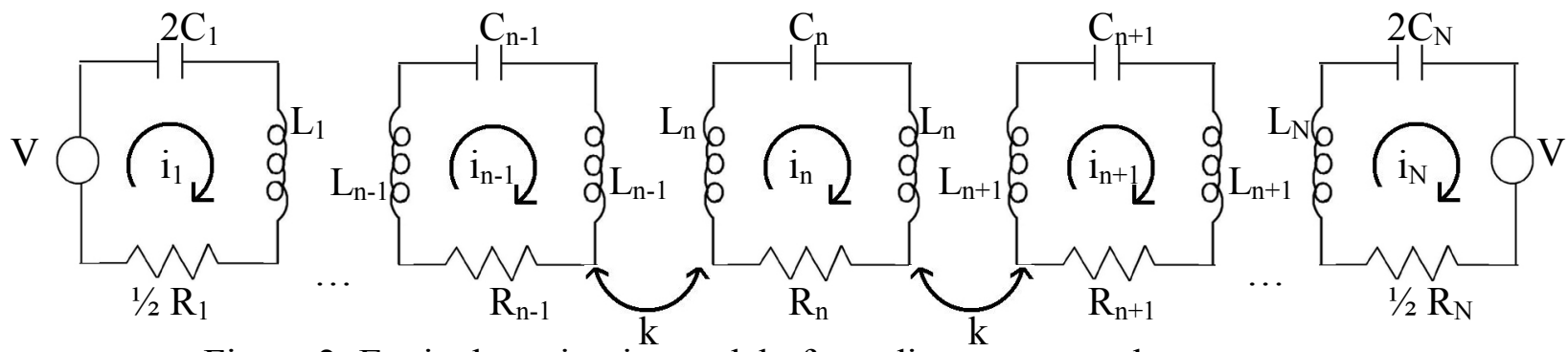

Figure 2: Equivalent circuits model of standing wave accelerator structure

Figure 1 depicts a general standing wave accelerator structure. Figure 2 depicts the equivalent circuits that model the accelerator structure with half cell boundary conditions. Each circuit consists of a resistor $\mathrm{R}$, an inductor $\mathrm{L}$, and a capacitor $\mathrm{C}$. The first and last circuits contain a voltage $\mathrm{V}$. The circuits have a coupling coefficient $\mathrm{k}$ that also represents the bandwidth of the structure. 
Applying Thevenin's equation for the network loop, the following circuit equations are obtained:

$$
\begin{aligned}
& V=i_{1}\left[j \omega L_{1}+\frac{1}{2} R_{1}+\frac{1}{j \omega 2 C_{1}}\right]+j \omega k \sqrt{L_{1} L_{2}} i_{2} \\
& 0=i_{n}\left[2 j \omega L_{n}+R_{n}+\frac{1}{j \omega 2 C_{n}}\right]+j \omega k \sqrt{L_{n}}\left[i_{n-1} \sqrt{L_{n-1}}+i_{n+1} \sqrt{L_{n+1}}\right] \\
& V=i_{N}\left[j \omega L_{N}+\frac{1}{2} R_{N}+\frac{1}{j \omega 2 C_{N}}\right]+j \omega k \sqrt{L_{N-1} L_{N}} i_{N-1}
\end{aligned}
$$

Further simplification gives the equations:

$$
\begin{aligned}
& \frac{V}{j \omega L_{1}}=i_{1}\left[1+\frac{\omega_{1}^{\prime}}{j \omega Q}-\frac{\omega_{1}^{2}}{\omega^{2}}\right]+\frac{k i_{2} \sqrt{L_{2}}}{\sqrt{L_{1}}} \\
& 0=2 i_{n}\left[2+\frac{2 \omega_{n}^{\prime}}{j \omega Q}-\frac{\omega_{n}^{2}}{\omega^{2}}\right]+\frac{k\left[i_{n-1} \sqrt{L_{n-1}}+i_{n+1} \sqrt{L_{n+1}}\right]}{\sqrt{L_{n}}} \\
& \frac{V}{j \omega L_{N}}=i_{N}\left[1+\frac{\omega_{N}^{\prime}}{j \omega Q}-\frac{\omega_{N}^{2}}{\omega^{2}}\right]+\frac{k i_{N-1} \sqrt{L_{N-1}}}{\sqrt{L_{N}}}
\end{aligned}
$$

where: $\mathrm{R}_{\mathrm{i}}=\frac{2 \omega_{\mathrm{i}}^{\prime} \mathrm{L}_{\mathrm{i}}}{\mathrm{Q}}, \omega_{\mathrm{i}}^{\prime}=\frac{1}{\sqrt{2 \mathrm{~L}_{\mathrm{i}} \mathrm{C}_{\mathrm{i}}}}, \mathrm{Q}$ is the quality factor, and $\omega_{\mathrm{I}}^{\prime}$ is the central frequency of each cell. For simplification purposes, $\omega_{i}^{2}=\omega_{i}^{\prime 2}\left[1+\frac{j \omega}{\omega_{i}^{\prime} Q}\right]$ and $X_{i}=i_{i} \sqrt{L_{i}}$. These substitutions yield the equations:

$$
\begin{aligned}
& \mathrm{X}_{1}+\mathrm{kX}_{2}=\frac{\omega_{1}^{2}}{\omega^{2}} \mathrm{X}_{1}+\frac{\mathrm{V}}{\mathrm{j} \omega \sqrt{\mathrm{L}_{1}}} \\
& \frac{\mathrm{kX} \mathrm{n}-1}{2}+\mathrm{X}_{\mathrm{n}}+\frac{\mathrm{kX}_{\mathrm{n}+1}}{2}=\frac{\omega_{\mathrm{n}}^{2}}{\omega^{2}} \mathrm{X}_{\mathrm{n}} \\
& \mathrm{kX}_{\mathrm{N}-1}+\mathrm{X}_{\mathrm{N}}=\frac{\omega_{\mathrm{N}}^{2}}{\omega^{2}} X_{\mathrm{N}}+\frac{\mathrm{V}}{\mathrm{j} \omega \sqrt{\mathrm{L}_{\mathrm{N}}}}
\end{aligned}
$$

These circuit equations may be represented in the following matrix form: 


$$
\left[\begin{array}{ccccc}
\frac{1}{\omega_{1}^{2}} & \frac{\mathrm{k}}{\omega_{1}^{2}} & 0 & \cdot & 0 \\
0 & & & & \cdot \\
\cdot & \frac{\mathrm{k}}{2 \omega_{\mathrm{n}}^{2}} & \frac{1}{\omega_{\mathrm{n}}^{2}} & \frac{\mathrm{k}}{2 \omega_{\mathrm{n}}^{2}} & \cdot \\
\cdot & & & 0 & 0 \\
0 & \cdot & 0 & \frac{\mathrm{k}}{\omega_{\mathrm{N}}^{2}} & \frac{1}{\omega_{\mathrm{N}}^{2}}
\end{array}\right]\left[\begin{array}{c}
\mathrm{X}_{1} \\
\cdot \\
\cdot \\
\cdot \\
\mathrm{X}_{\mathrm{N}}
\end{array}\right]=\frac{1}{\omega^{2}}\left[\begin{array}{c}
\mathrm{X}_{1} \\
\cdot \\
\cdot \\
\cdot \\
\mathrm{X}_{\mathrm{N}}
\end{array}\right]
$$

As with the 3-cell model (See Appendix A), the eigenvectors and eigenvalues (and subsequent eigenfrequencies) were calculated for the 15-cell model.

Both the $\pi$ mode and $\pi / 2$ mode of acceleration were investigated for the X-band structure with coupling coefficient $\mathrm{k}$ of $2.5 \%$ and $4 \%$. The coupling coefficient is equivalent to the bandwidth of the frequencies. The modal Q, eigenfrequencies, and distances to the next two nearest modes were found. The $\pi$ mode was then further investigated. Random errors on the order of one megahertz were added to the central frequency of each cell for the $\pi$ mode of operation. Tuning of the random errors was then accomplished by writing code to minimize the root mean square of the deviation of amplitudes from the central cell of the 15th mode by changing the central frequency of the first, central, and last cells. The random errors were introduced and tuned over 50 trials. Systematic errors of $0.5 \mathrm{MHz}, 1.0 \mathrm{MHz}, 2.0 \mathrm{MHz}$, and 3.0 MHz were also added to the central frequency of each cell for the $\pi$ mode of operation. Tuning of the systematic errors required a different method because the $15^{\text {th }}$ mode remains flat after the introduction of the error. The first and last cells were tuned by $-\frac{\Delta{\mathrm{n} \delta \mathrm{F}_{0}}_{2}}{2}$, and the center cell was tuned by $-\Delta \mathrm{n} \delta \mathrm{F}_{0}$, where $\Delta \mathrm{n}$ is the distance (in number of cells) to the next tuned cell and $\delta \mathrm{F}_{0}$ is the amount of introduced systematic error. 


\subsection{Wakefields in Standing Wave and Traveling Wave Structures}

A wakefield results from a wave scattered by boundary discontinuities and produces image currents set up on the walls of the accelerator. The integrated effect of wakefields can be partitioned into longitudinal and transverse wake potentials. Of particular interest is the transverse wake potential, a cause of BBU, given by the equation:

$$
\overrightarrow{\mathrm{W}}_{\perp}\left(\overrightarrow{\mathrm{r}}, \overrightarrow{\mathrm{r}}^{\prime}, \mathrm{s}\right)=\frac{1}{\mathrm{q}} \int_{\mathrm{z}_{1}}^{\mathrm{z}_{2}} \mathrm{dz}\left[\overrightarrow{\mathrm{E}}_{\perp}+\mathrm{c}(\hat{\mathrm{z}} \times \vec{\beta})\right]_{\mathrm{t}=(\mathrm{z}+\mathrm{s}) / \mathrm{c}}
$$

where $\vec{r}$ and $\vec{r}$ are the transverse offsets of the driving and test charges from the $z$ axis, $s$ is the distance behind point charge $\mathrm{q}, \overrightarrow{\mathrm{E}}_{\perp}$ is the transverse electric field, c is the speed of light, $\hat{z}$ is a unit vector in the direction of motion of both the driving and test charges (assumed to be along the $\mathrm{z}$ axis), and $\vec{\beta}$ is the magnetic field. The driving charge is assumed to enter the cavity structure at $\mathrm{z}, \mathrm{t}=0$ and to exit at $\mathrm{z}=$ the length of the accelerator. The test particle enters and leaves the cavity at $z_{1}$ and $z_{2}$. Synchronous frequencies and kick factors of five cells over six bands of the dipole wakefield were given for a $2 \pi / 3$ mode 206 -cell traveling wave structure and a $\pi$ mode 120-cell standing wave structure. The remaining synchronous frequencies and kick factors were interpolated. The wakefield equation as a function of $s$, the distance behind the first bunch, is as follows:

$$
\text { wakefield (s) }=\frac{2}{N}\left|\sum_{n=1}^{N} K_{n} e^{\frac{-\omega_{n} s}{2 Q_{n}} \mathrm{c}} e^{\frac{j \omega_{n} s}{c}}\right|
$$

$\mathrm{K}_{\mathrm{n}}$ represents the kick factors corresponding to each cell, $\omega_{\mathrm{n}}$ represents the angular frequency, and $\mathrm{Q}_{\mathrm{n}}$ represents the quality factor. The interpolated synchronous frequencies and kick factors were used to calculate the first six bands of the dipole wakefield in both the standing wave and traveling wave structures. 


\subsection{Results}

\subsection{Tuning of Modes for the Standing Wave Structure}

Table 1 shows the modal $\mathrm{Q}$ for the $\pi$ and $\pi / 2$ modes of operation with a coupling coefficient $\mathrm{k}$ of $2.5 \%$ and $4.0 \%$ for a 15 -cavity standing wave structure.

\begin{tabular}{|c|c|c|c|c|}
\hline & & \multicolumn{3}{|c|}{ modal Q } \\
\cline { 3 - 5 } mode & $\mathrm{k}$ & cell 1 & cell 8 & cell 15 \\
\hline$\pi$ & 0.025 & 7086.96 & 7000 & 6911.95 \\
\hline$\pi$ & 0.04 & 7138.63 & 7000 & 6858.57 \\
\hline$\pi / 2$ & 0.025 & 7086.96 & 7000 & 6911.95 \\
\hline$\pi / 2$ & 0.04 & 7138.63 & 7000 & 6858.57 \\
\hline
\end{tabular}

Table 1: Modal Q at different modes of operation and coupling coefficients

Table 2 shows the eigenfrequencies for the $\pi$ mode of operation with a coupling coefficient $\mathrm{k}$ of $2.5 \%$ and $4.0 \%$ for a 15 -cavity standing wave structure.

\begin{tabular}{|c|c|c|c|c|c|}
\hline \multirow{2}{*}{$\mathrm{k}$} & \multicolumn{5}{|c|}{$\pi$ mode Eigenfrequencies $(\mathrm{GHz})$} \\
\cline { 2 - 6 } & cell 1 & cell 8 & cell 13 & cell 14 & cell 15 \\
\hline 0.025 & 11.142 & 11.28 & 11.41 & 11.42 & 11.424 \\
\hline 0.04 & 10.976 & 11.193 & 11.401 & 11.418 & 11.424 \\
\hline
\end{tabular}

Table 2: Eigenfrequencies for $\pi$ mode of operation

Table 3 shows the eigenfrequencies for the $\pi / 2$ mode of operation with a coupling coefficient $\mathrm{k}$ of $2.5 \%$ and $4.0 \%$ for a 15 -cavity standing wave structure.

\begin{tabular}{|c|c|c|c|c|c|c|c|}
\hline \multirow{2}{*}{$\mathrm{k}$} & \multicolumn{7}{|c|}{$\pi / 2$ mode Eigenfrequencies $(\mathrm{GHz})$} \\
\cline { 2 - 8 } & cell 1 & cell 6 & cell 7 & cell 8 & cell 9 & cell 10 & cell 15 \\
\hline 0.025 & 11.284 & 11.363 & 11.392 & 11.424 & 11.456 & 11.487 & 11.57 \\
\hline 0.04 & 11.202 & 11.326 & 11.374 & 11.424 & 11.475 & 11.524 & 11.66 \\
\hline
\end{tabular}

Table 3: Eigenfrequencies for $\pi / 2$ mode of operation

Table 4 shows the distance to the next two nearest modes for the $\pi$ and $\pi / 2$ modes of operation with a coupling coefficient $\mathrm{k}$ of $2.5 \%$ and $4.0 \%$. 


\begin{tabular}{|c|c|c|c|c|c|}
\hline mode & $\mathrm{k}$ & \multicolumn{2}{|l|}{$\begin{array}{l}\text { Distance to next nearest } \\
\text { mode (MHz) }\end{array}$} & $\begin{array}{l}\text { Distance to second } \\
\text { nearest mode (MHz) }\end{array}$ \\
\hline$\pi$ & 0.025 & \multicolumn{2}{|c|}{3.67033} & \multicolumn{2}{|c|}{14.4767} \\
\hline$\pi$ & 0.04 & \multicolumn{2}{|c|}{5.96249} & \multicolumn{2}{|c|}{23.4967} \\
\hline$\pi / 2$ & 0.025 & 31.644 & 31.9092 & 61.4591 & 62.4673 \\
\hline$\pi / 2$ & 0.04 & 50.5047 & 51.1835 & 97.8618 & 100.443 \\
\hline
\end{tabular}

Table 4: Distance to next nearest and second next nearest modes

Random errors with a root mean square of $1 \mathrm{MHz}$ were generated during each trial run for the $\pi$ mode of operation. Table 5 depicts the average root mean square of deviation from the central cell in the $15^{\text {th }}$ mode for 50 trial runs:

\begin{tabular}{|c|c|c|}
\hline \multirow{2}{*}{$\mathrm{k}$} & \multicolumn{2}{|c|}{ RMS } \\
\cline { 2 - 3 } & $\begin{array}{c}\text { before } \\
\text { correction }\end{array}$ & $\begin{array}{c}\text { after } \\
\text { correction }\end{array}$ \\
\hline 0.025 & $1.86 \%$ & $0.36 \%$ \\
\hline 0.04 & $1.03 \%$ & $0.21 \%$ \\
\hline
\end{tabular}

Table 5: Average RMS of 50 trials for $\pi$ mode

Table 6 shows the average values of the cell frequencies after tuning for 50 trial runs:

\begin{tabular}{|c|c|c|c|c|c|c|}
\hline \multirow{2}{*}{$\mathrm{k}$} & \multicolumn{3}{|c|}{ Initial Frequency $(\mathrm{GHz})$} & \multicolumn{3}{c|}{ Frequency after tuning $(\mathrm{GHz})$} \\
\cline { 2 - 7 } & cell 1 & cell 8 & cell 15 & cell 1 & cell 8 & cell 15 \\
\hline 0.025 & 11.142 & 11.28 & 11.424 & 11.1419 & 11.2803 & 11.424 \\
\hline 0.04 & 10.976 & 11.193 & 11.424 & 10.9759 & 11.1932 & 11.424 \\
\hline
\end{tabular}

Table 6: Average frequencies of tuned cells for $\pi$ mode

The following results correspond to one representative trial run with random errors. The following errors (in $\mathrm{MHz}$ ) were introduced to each respective cell: $-1.44,1.15,0.4,-1.01,-0.62$, $0.89,0.45,-0.23,-0.66,1.08,-1.19,0.78,1.57,1.05,0.14$. Figure 3 displays the dispersion curves for cells with a bandwidth (coupling coefficient) of $2.5 \%$ at the $\pi$ mode of operation: 

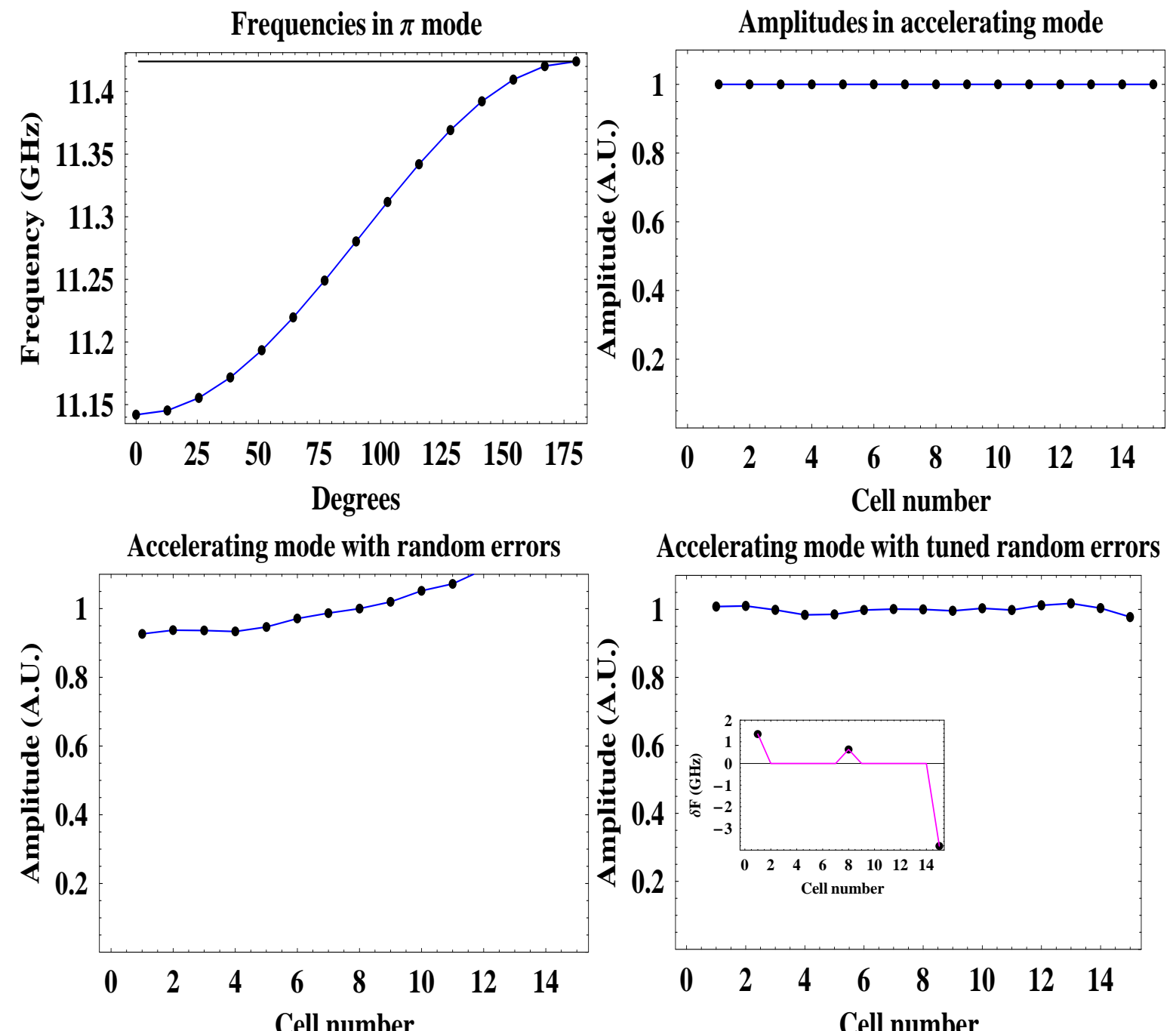

Accelerating mode with tuned random errors

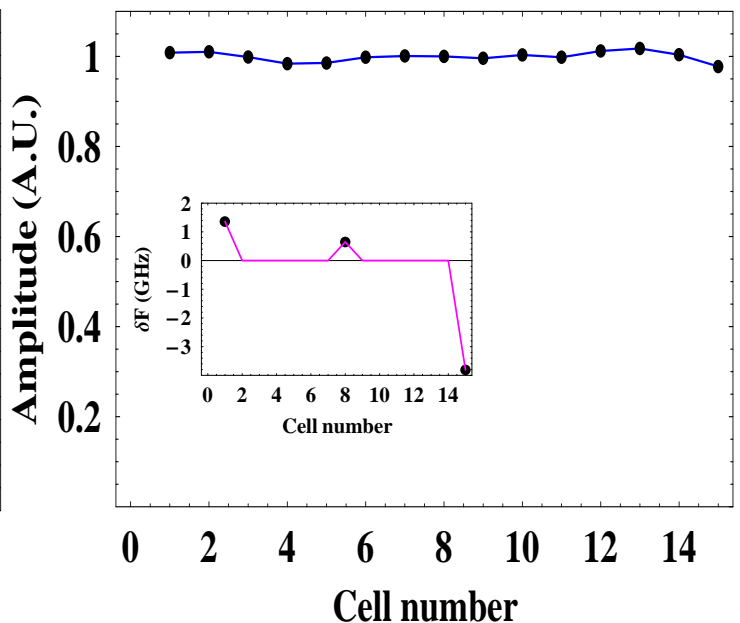

Figure 3: Dispersion curves at $\pi$ mode of acceleration and $2.5 \%$ bandwidth

The root mean square of deviation was minimized from $2.20 \%$ to $0.27 \%$ by tuning the $1^{\text {st }}$ cell by $1.36 \mathrm{MHz}$, the $8^{\text {th }}$ cell by $0.65 \mathrm{MHz}$, and the $15^{\text {th }}$ cell by $-3.81 \mathrm{MHz}$. The tuning required is shown inset to Figure 3.

Systematic errors of $0.5 \mathrm{MHz}, 1.0 \mathrm{MHz}, 2.0 \mathrm{MHz}$, and $3.0 \mathrm{MHz}$ were introduced to the $\pi$ mode of acceleration for coupling coefficient $\mathrm{k}$ of $2.5 \%$ and $4.0 \%$. Table 7 displays the frequencies and the root mean square of deviation for the accelerating mode: 


\begin{tabular}{|c|c|c|c|c|}
\hline \multirow[b]{2}{*}{$\mathrm{k}$} & \multirow[b]{2}{*}{$\begin{array}{l}\text { error } \\
(\mathrm{MHz})\end{array}$} & \multicolumn{2}{|c|}{$\begin{array}{c}\text { Frequency at } \\
\text { accelerating mode }(\mathrm{GHz})\end{array}$} & \multirow[b]{2}{*}{$\begin{array}{l}\text { RMS after } \\
\text { correction }\end{array}$} \\
\hline & & $\begin{array}{l}\text { before } \\
\text { correction }\end{array}$ & $\begin{array}{c}\text { after } \\
\text { correction }\end{array}$ & \\
\hline 0.025 & 0.5 & 11.4245 & 11.4241 & $1.11 \%$ \\
\hline 0.025 & 1 & 11.425 & 11.4243 & $2.12 \%$ \\
\hline 0.025 & 2 & 11.426 & 11.4247 & $3.92 \%$ \\
\hline 0.025 & 3 & 11.427 & 11.4251 & $5.45 \%$ \\
\hline 0.04 & 0.5 & 11.4245 & 11.4241 & $0.70 \%$ \\
\hline 0.04 & 1 & 11.425 & 11.4243 & $1.36 \%$ \\
\hline 0.04 & 2 & 11.426 & 11.4246 & $2.58 \%$ \\
\hline 0.04 & 3 & 11.4271 & 11.425 & $3.68 \%$ \\
\hline
\end{tabular}

Table 7: Results from minimizing systematic error for $\pi$ mode

Figure 4 depicts the accelerating mode for the $\pi$ mode of operation with $2.5 \%$ bandwidth.

Accelerating mode with tuned systematic errors

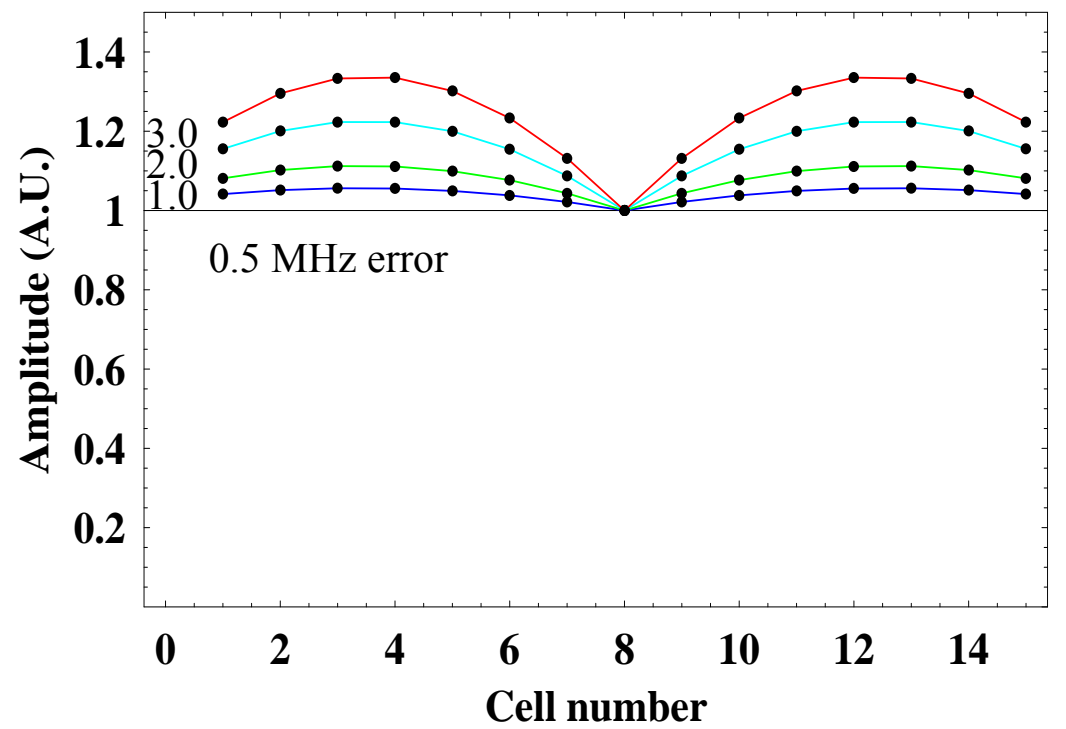

Figure 4: Accelerating mode after minimizing errors

Figure 5 displays the dispersion curves for cells with a bandwidth (coupling coefficient) of $2.5 \%$ at the $\pi$ mode of operation and a systematic error of $3.0 \mathrm{MHz}$ : 

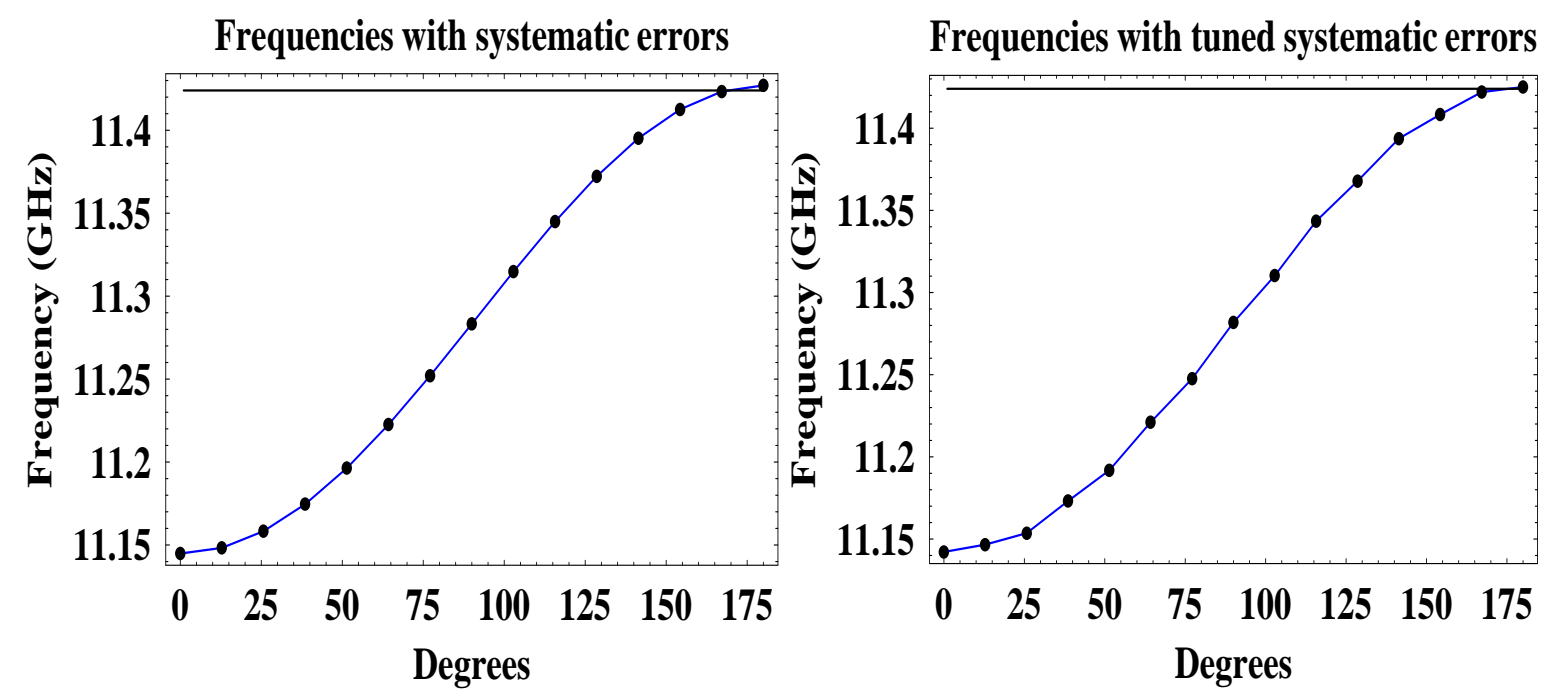

Accelerating mode with systematic errors
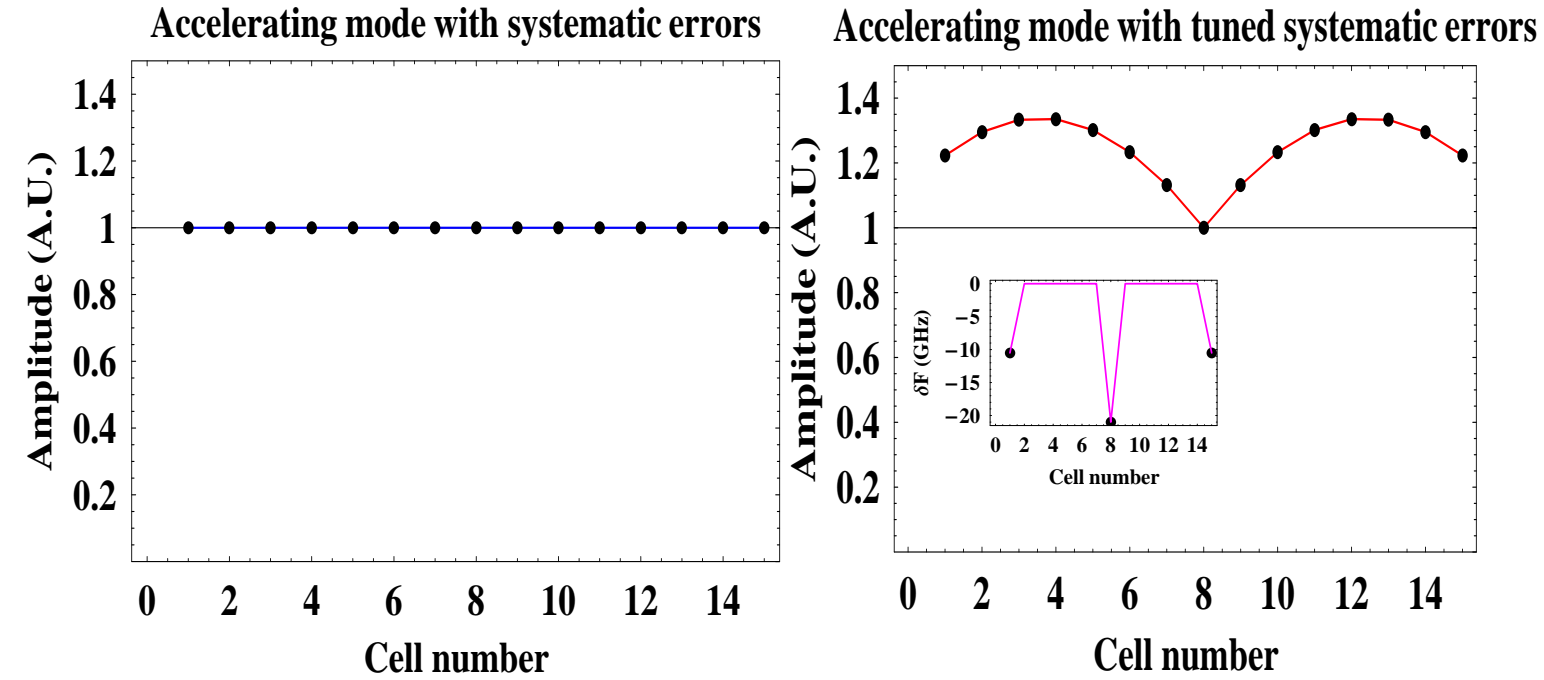

Figure 5: Dispersion curves at $\pi$ mode of acceleration and 2.5\% bandwidth with $3.0 \mathrm{MHz}$ error The accelerating frequency was tuned from $11.427 \mathrm{GHz}$ to $11.4251 \mathrm{GHz}$ by adjusting the $1^{\text {st }}$ cell by $-10.5 \mathrm{MHz}$, the $8^{\text {th }}$ cell by $-21 \mathrm{MHz}$, and the $15^{\text {th }}$ cell by $-10.5 \mathrm{MHz}$. The tuning required is shown inset to Figure 5.

\subsection{Wakefields in Standing Wave and Traveling Wave Structures}

The wakefield causes the beam to become unstable; for this reason, the band structure was investigated. Figure 6 and Figure 7 depict the kick factors corresponding to the first six bands of the dipole wakefield in the standing wave structure and the traveling wave structure: 

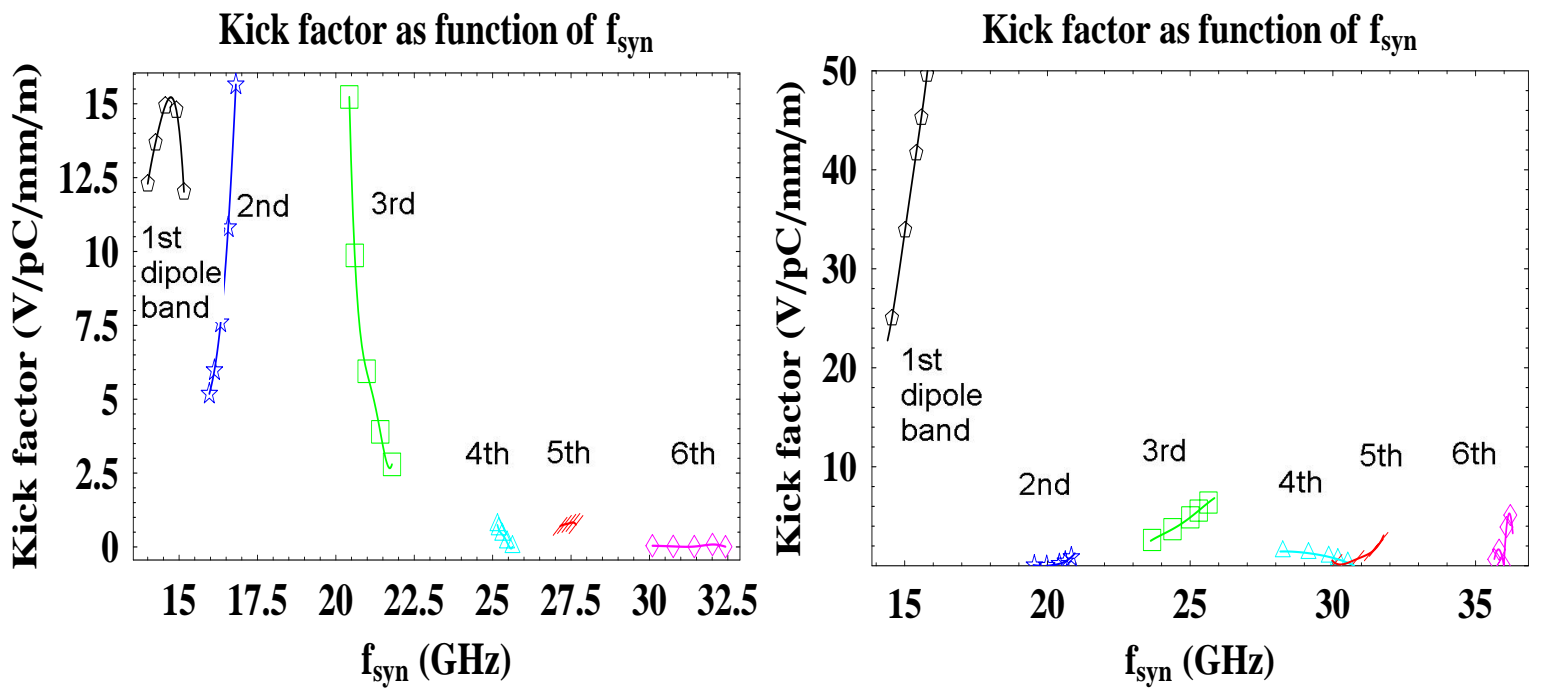

Figure 6: Standing Wave structure

Figure 7: Traveling Wave structure

Figure $8 \mathrm{a}$ and Figure $8 \mathrm{~b}$ depict the first six bands of the dipole wakefield with respect to the distance behind the first accelerating bunch in the standing wave structure and the traveling wave structure. The points represent the accelerated bunches, separated by a $0.42 \mathrm{~mm}$ spacing.
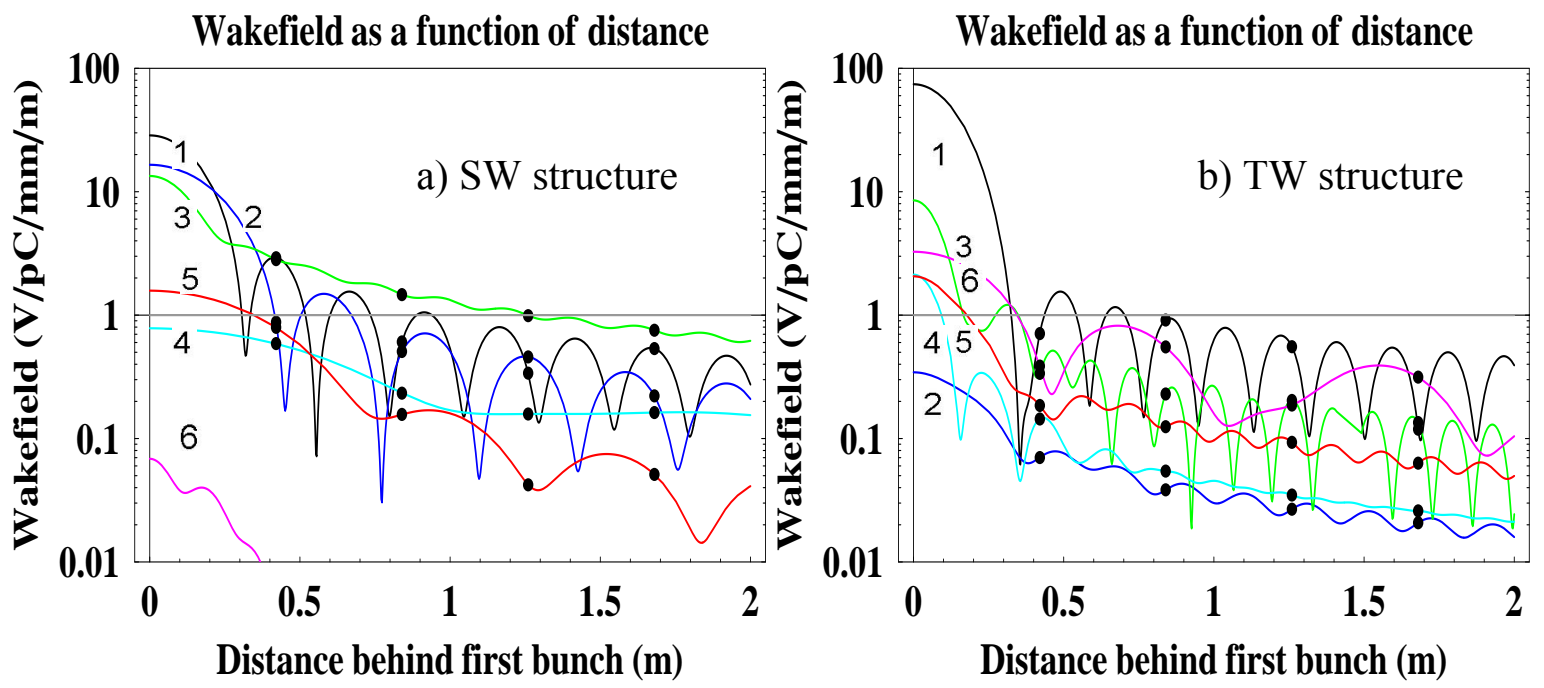

Figure 8: Comparison of Envelope of Dipole Wakefield Bands

Figure $9 \mathrm{a}$ and Figure $9 \mathrm{~b}$ depict the relative magnitudes of each band by displaying the sums of the first six bands of the dipole wakefield with respect to the distance behind the first accelerating bunch in the standing wave structure and the traveling wave structure: 

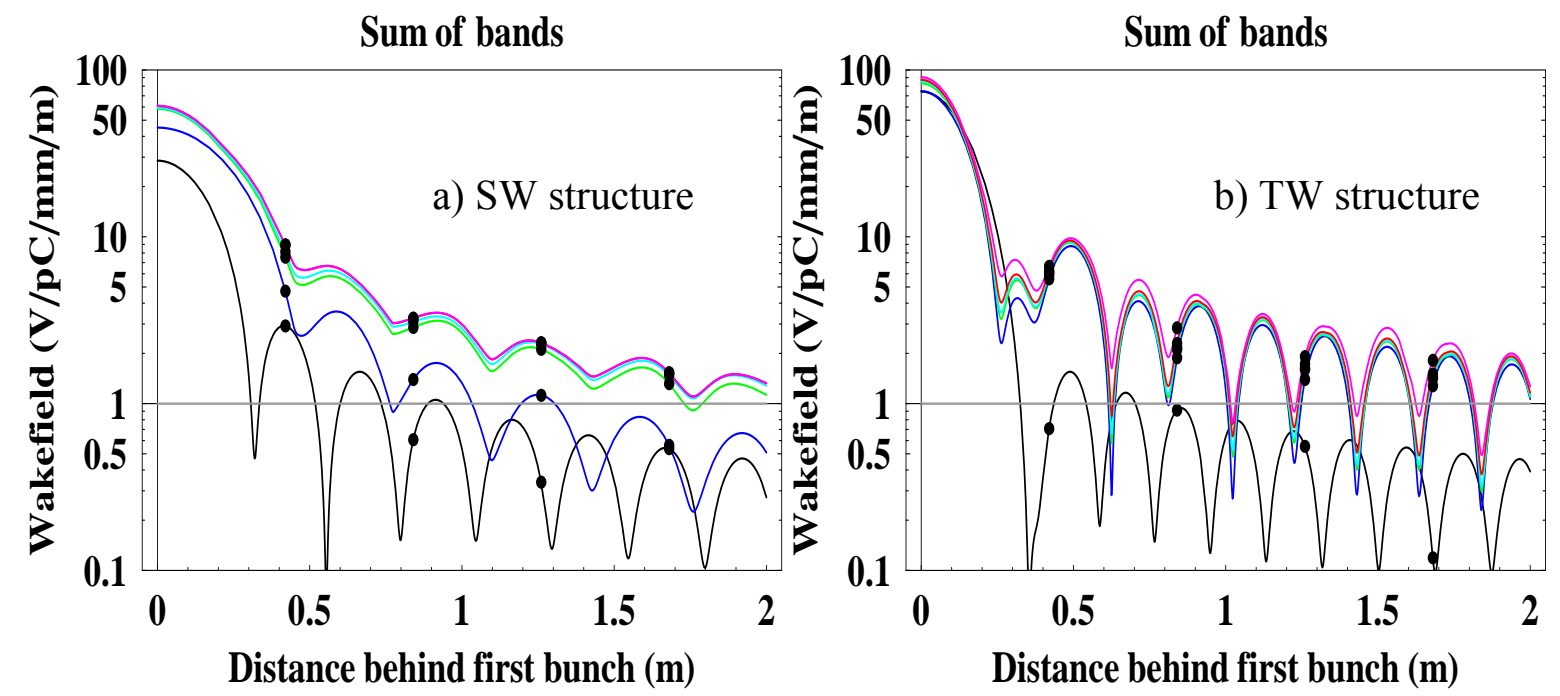

Figure 9: Comparison of Sum of the Envelope of the Wakefield Bands

\subsection{Discussion and Conclusions}

\subsection{Tuning of Modes for the Standing Wave Structure}

As seen in Table 1, the modal Q values are on the order of the initial quality factor (7000) for the cells. The modal Q values are the same for each coupling coefficient regardless of the mode of operation. The coupling coefficient is a small quantity and is a second order effect which does not change the $\mathrm{Q}$. As a result, the $\mathrm{Q}$ for a particular mode is relatively constant.

Table 2 shows that the eigenfrequency of the last cell (cell 15) in the $\pi$ mode is 11.424 , the Xband frequency in the accelerator. Table 3 shows the eigenfrequency of the middle cell (cell 8) in the $\pi / 2$ mode is 11.424 .

Table 4 presents the distance from the accelerating mode to the next two nearest modes. Since excitation for the standing wave structure originates at the center, the next excited mode is two modes away from the current mode (See Appendix A). It is therefore important to know the 
distance from the accelerating mode to the $2^{\text {nd }}$ to next mode. Table 4 clearly indicates that the nearest modes to the $\pi$ mode are much closer than the nearest modes to the $\pi / 2$ mode. The second to nearest mode to the $\pi$ mode with $2.5 \%$ coupling is around $14 \mathrm{MHz}$ while the second to nearest mode to the $\pi / 2$ mode with $2.5 \%$ coupling is around $62 \mathrm{MHz}$. These results show that acceleration under the $\pi$ mode of operation is more sensitive to errors than acceleration under the $\pi / 2$ mode.

As seen in Table 5 , in the $\pi$ mode of operation, the deviation resulting from introduced random errors was greater for the lower coupling coefficient. A smaller coupling coefficient k indicates a weaker coupling between the cavities of the accelerator. Thus, the smaller the coupling, the more sensitive the structure is to errors.

From Figure 3, it is clear that the introduced random errors did not have much of an effect on the frequencies but had a great effect on the flatness of the field of the accelerating mode. Tuning the $1^{\text {st }}, 8^{\text {th }}$, and $15^{\text {th }}$ cell by $1.36 \mathrm{MHz}, 0.65 \mathrm{MHz}$, and $-3.81 \mathrm{MHz}$, respectively, minimized the root mean square of deviation from $2.20 \%$ to $0.27 \%$. Figure 5 shows that the opposite is true for systematic errors. The systematic errors effected a drastic shift in the frequencies while the field of the accelerating mode remained flat. In the case of the $\pi$ mode of operation with a bandwidth of $2.5 \%$ and a systematic error of $3.0 \mathrm{MHz}$, tuning the $1^{\text {st }}, 8^{\text {th }}$, and $15^{\text {th }}$ cell by $-10.5 \mathrm{MHz},-21$ $\mathrm{MHz}$, and -10.5 MHz, respectively, adjusted the accelerating frequency from $11.427 \mathrm{GHz}$ to $11.4251 \mathrm{GHz}$. 
Table 7 and Figure 4 show that the root mean square of deviation increases with the amount of introduced systematic error. It is also important to note from Table 7 that the coefficient of coupling is crucial to the flatness of the field of the accelerating mode after tuning. The smaller the coupling, the larger the root mean square of deviation is after tuning.

This investigation of the $\pi$ and $\pi / 2$ modes of operation used the nearest neighbor coupling model. Future work could include studying the $\pi$ and $\pi / 2$ modes of operation with the next nearest neighbor coupling model. Since the $\pi / 2$ mode of operation has a larger margin for error than the $\pi$ mode has, future research could investigate the field stability of the $\pi / 2$ mode of operation.

\subsection{Wakefields in Standing Wave and Traveling Wave Structures}

As seen in Figure 6, the kick factors are significantly higher for the first three dipole bands than the other bands of the standing wave structure. In the traveling wave structure, the kick factor is significantly higher for the first dipole band than the other bands. Figure 8 displays the wakefields for both the standing wave and traveling wave structure. Beam Break Up occurs above a $1 \mathrm{~V} / \mathrm{pC} / \mathrm{mm} / \mathrm{m}$ threshold. It is clear that the first, second, third, fourth, and fifth bands of the dipole wakefield for the standing wave structure are cause for concern while the first, third, and sixth bands of the dipole wakefield for the traveling wave structure are cause for concern.

Notice that in Figure 9a, the first witness bunch resides on a node (maximum) for the first band. The effect of the wakefield is maximized at the node. In order to minimize the effect of the wakefield, the cavities can be tuned so that the first witness bunch sits at an antinode instead of a node. 
Tuning occurs by changing the dimensions of the cavity. The dimensions can be changed by reducing the iris in a smooth adiabatic fashion and changing the thickness of the iris in a similar manner. Damping is also needed because the wakefield eventually recoheres and rises rapidly at this point.

\subsection{Acknowledgements}

I thank the United States Department of Energy- Office of Science for sponsoring the Energy Research Undergraduate Laboratory Fellowship program. I give many thanks to my mentor Dr. Roger M. Jones for his guidance throughout my research. My thanks go to Dr. Helen Quinn and Dr. Sekazi Mtingwa for organizing and directing this program. I also thank the Stanford Linear Accelerator Center for allowing me the use of its facilities to perform my research.

\subsection{Appendix A}

The following diagram depicts equivalent circuits to three coupled standing wave cavities.

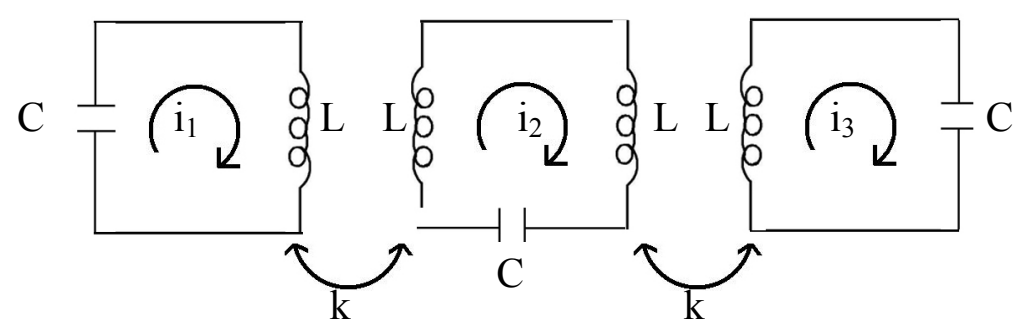

Figure 10: Three Coupled Standing Wave Cavities

Each circuit consists an inductor L and a capacitor C. The central cavity has two inductors. All the inductors and capacitors are equal. Assume that voltage $V$ of the first circuit is equal to 0 . The circuits have a coupling coefficient $\mathrm{k}$. 
The derived circuit equations are as follows:

$$
\begin{aligned}
& 0=i_{1}\left[j \omega L+\frac{1}{j \omega 2 L}\right]+j \omega \mathrm{ki}_{2} \\
& 0=i_{2}\left[2 j \omega L+\frac{1}{j \omega C}\right]+j \omega \mathrm{kLi}_{1}+j \omega k \mathrm{Li}_{3} \\
& 0=\mathrm{i}_{3}\left[j \omega \mathrm{L}+\frac{1}{j \omega 2 \mathrm{~L}}\right]+j \omega \mathrm{kLi}_{2}
\end{aligned}
$$

The central frequency $\omega_{0}$ is given by the equation $\omega_{0}=\frac{1}{\sqrt{2 \mathrm{LC}}}$. If the circuit equations are normalized by L, it follows that:

$$
\begin{aligned}
& 0=i_{1} j \omega\left[1-\frac{\omega_{0}^{2}}{\omega^{2}}\right]+j \omega \mathrm{ki}_{2} \\
& 0=2 \mathrm{i}_{2} \mathrm{j} \omega\left[1-\frac{\omega_{0}^{2}}{\omega^{2}}\right]+\mathrm{i}_{1} \mathrm{j} \omega \mathrm{k}+\mathrm{i}_{3} \mathrm{j} \omega \mathrm{k} \\
& 0=\mathrm{i}_{3} \mathrm{j} \omega\left[1-\frac{\omega_{0}^{2}}{\omega^{2}}\right]+\mathrm{j} \omega \mathrm{ki}_{2}
\end{aligned}
$$

For simplification purposes, $X_{i}=i_{i} j \omega$. Substitution yields:

$$
\begin{aligned}
& \mathrm{X}_{1}+\mathrm{kX}_{2}=\frac{\omega_{0}^{2}}{\omega^{2}} \mathrm{X}_{1} \\
& \frac{\mathrm{k}}{2} \mathrm{X}_{1}+\mathrm{X}_{2}+\frac{\mathrm{k}}{2} \mathrm{X}_{3}=\frac{\omega_{0}^{2}}{\omega^{2}} \mathrm{X}_{2} \\
& \mathrm{kX}_{2}+\mathrm{X}_{3}=\frac{\omega_{0}^{2}}{\omega^{2}} \mathrm{X}_{3}
\end{aligned}
$$

The circuit equations can be written in matrix form:

$$
\left[\begin{array}{ccc}
1 & \mathrm{k} & 0 \\
\frac{\mathrm{k}}{2} & 1 & \frac{\mathrm{k}}{2} \\
0 & \mathrm{k} & 1
\end{array}\right]\left[\begin{array}{l}
\mathrm{X}_{1} \\
\mathrm{X}_{2} \\
\mathrm{X}_{3}
\end{array}\right]=\frac{\omega_{0}^{2}}{\omega^{2}}\left[\begin{array}{l}
\mathrm{X}_{1} \\
\mathrm{X}_{2} \\
\mathrm{X}_{3}
\end{array}\right]
$$


The eigenvalues and eigenvectors of the matrix can then be calculated. The eigenfrequencies can be calculated from the eigenvalues of the matrix with the relationship eigenfrequency $=\sqrt{\frac{1}{\text { eigenvalue }}}$. The eigenfrequencies are the frequencies of maximum oscillation (resonance frequencies).

The eigenvectors describe the modes of the structure. For a three-cell structure, there are three modes: $0, \pi / 2$, and $\pi$. The following graphs depict the $0, \pi / 2$, and $\pi$ modes when the coupling coefficient is $2.5 \%$.
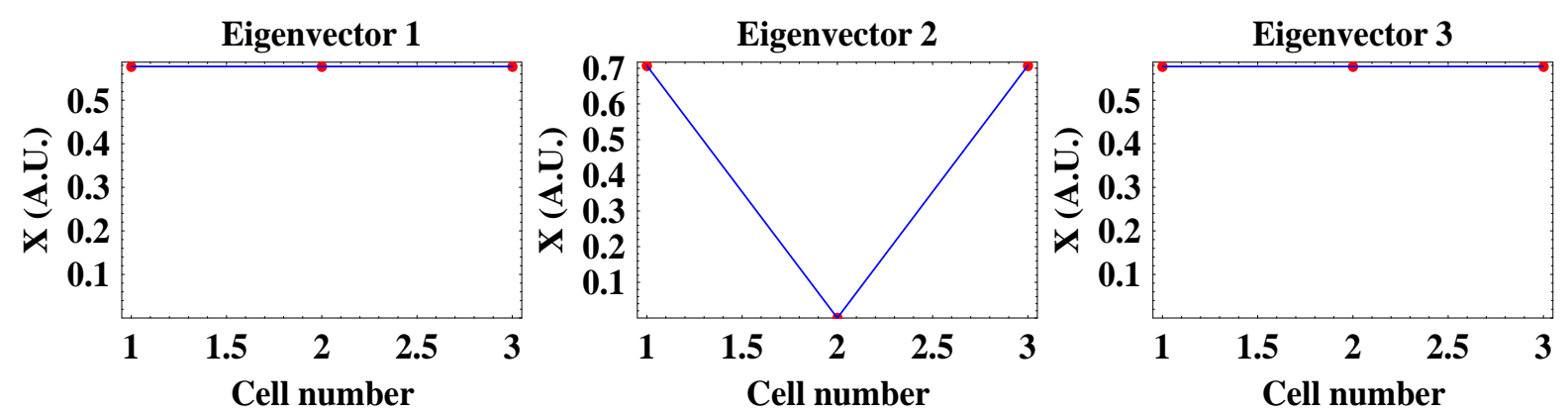

Figure 11: Modes of a 3-cell Standing Wave structure

In the 0 mode, all the cavities are oscillating together, so the mode appears flat without phase. In the $\pi / 2$ mode, the middle cavity is not excited. Plotting of modes for circuits of different lengths indicate that central mode occurs at a maximum for every other eigenvector. Subsequently, the central mode also occurs at a minimum for every other eigenvector. In the $\pi$ mode, every other mode is in phase. However, the project expands this simple circuit to 15 cells and includes resistance. Since resistance is included, the quality factor Q introduces an imaginary component to the frequency. Thus, the absolute value of the $\pi$ mode is plotted, and the amplitudes appear flat. 


\subsection{Appendix B}

The following diagram depicts equivalent circuits to two coupled standing wave cavities.

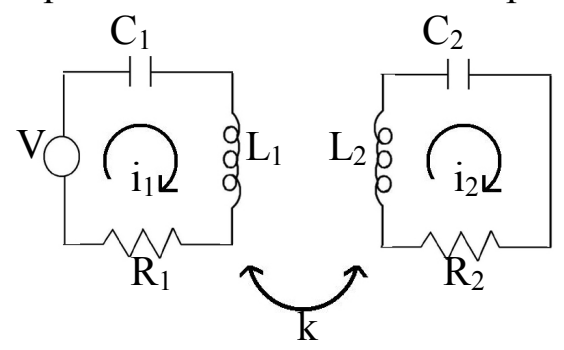

Figure 12: Two Coupled Standing Wave Cavities

Each circuit consists of a resistor $\mathrm{R}$, an inductor $\mathrm{L}$, and a capacitor $\mathrm{C}$. The first circuit contains a voltage $\mathrm{V}$. The circuits have a coupling coefficient $\mathrm{k}$.

Applying Thevenin's network equation, the following circuit equations are obtained:

$$
\begin{aligned}
& V_{1}=i_{1}\left[R_{1}+j \omega L_{1}+\frac{1}{j \omega C_{1}}\right]+j \omega k \sqrt{L_{1} L_{2}} i_{2} \\
& 0=V_{2}=i_{2}\left[R_{2}+j \omega L_{1}+\frac{1}{j \omega C_{2}}\right]+j \omega k \sqrt{L_{1} L_{2}} i_{1}
\end{aligned}
$$

Equation 1 can then be rewritten in terms of $i_{1}$ :

$$
V_{1}=i_{1}\left[R_{1}+j \omega L_{1}+\frac{1}{j \omega C_{1}}\right]+\frac{\omega^{2} k^{2} L_{1} L_{2} i_{1}}{R_{2}+j \omega L_{1}+\frac{1}{j \omega C_{2}}}
$$

The quality factor $Q$ is given by $Q_{n}=\frac{\omega_{n} L_{n}}{R_{n}}$ and $\omega_{n}^{2}=\frac{1}{L_{n} C_{n}}$. After careful substitution, the impedance / inductance of the first circuit is found to be:

$$
\overline{\mathrm{z}}_{1}=\frac{\mathrm{z}_{1}}{\mathrm{~L}_{1}}=\frac{\mathrm{V}_{1}}{\mathrm{i}_{1} \mathrm{~L}_{1}}=\frac{\omega_{1}}{\mathrm{Q}_{1}}+j \omega\left[1-\frac{\omega_{1}^{2}}{\omega^{2}}\right]+\frac{\omega^{2} \mathrm{k}^{2}}{\frac{\omega_{2}}{\mathrm{Q}_{2}}+\mathrm{j} \omega\left[1-\frac{\omega_{2}^{2}}{\omega^{2}}\right]}
$$


This impedance (normalized to the inductance) may be graphed as a function of angular frequency $\omega(=2 \pi f)$. For our purposes, we will graph the inverse of $\bar{z}_{1}$ (inductance / impedance) as a function of frequency $\mathrm{f}$. The following graphs depict $\overline{\mathrm{Z}}_{1}$ with an infinite $\mathrm{Q}_{1}$ and a $\mathrm{Q}_{2}$ of 100 , 50,20 , and 10 .
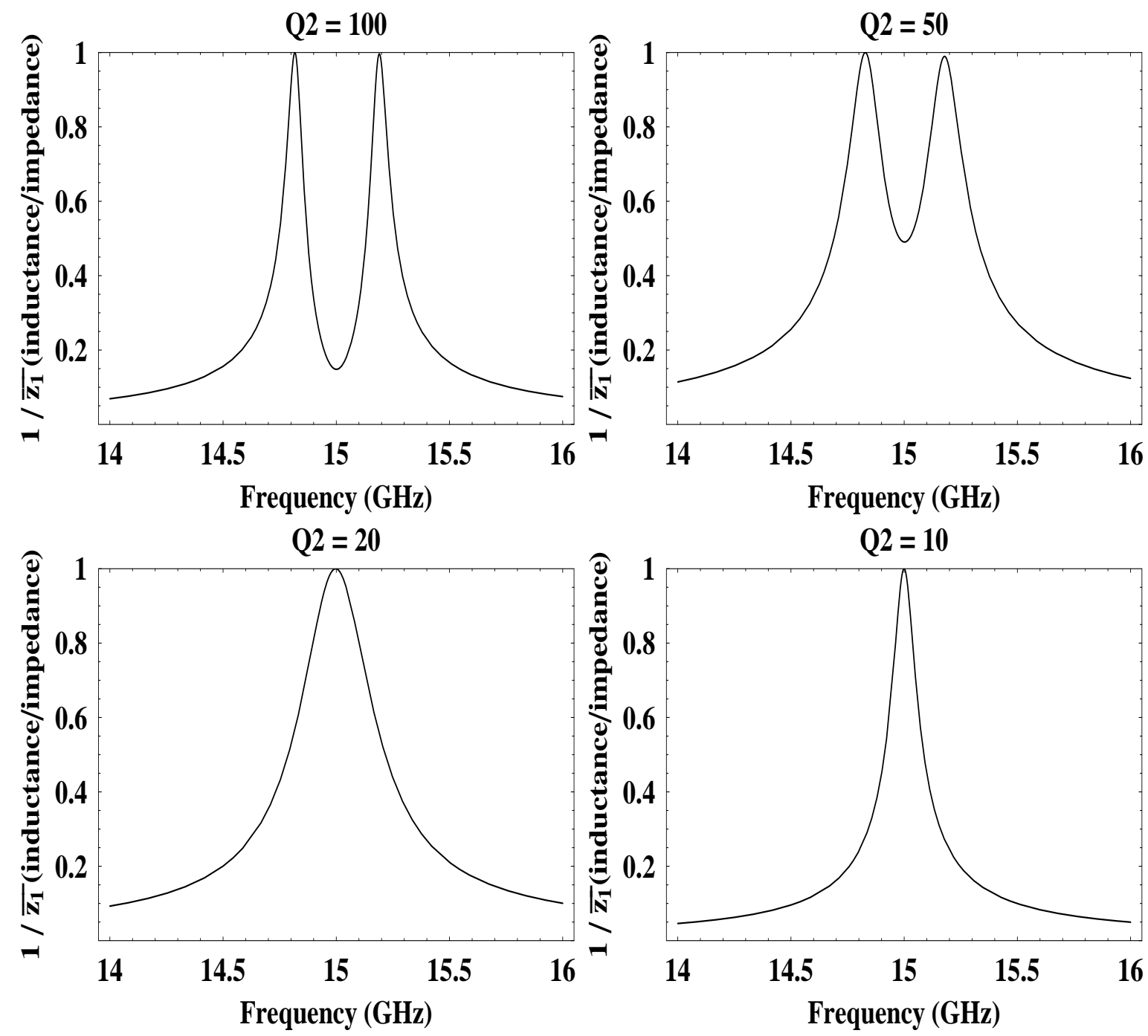

Figure 13: Dispersion curves at different Quality Factors

Note that when $\mathrm{Q}_{2}$ is high, peaks occur at two different frequencies. The peaks correspond to the central frequencies of the two separate cavities. As $\mathrm{Q}_{2}$ decreases, the peaks become less distinct, 
eventually consolidating into one peak. $B y \mathrm{Q}_{2}=20$, only one maximum remains; altering one cavity will tune the other cavity as well.

The modal Q is found by dividing central frequency by bandwidth. The bandwidth is measured from points residing at $1 / 2$ the height of the maximum. The following table presents the calculated modal Q values for the four graphs:

\begin{tabular}{|c|c|c|}
\hline $\mathrm{Q}_{2}$ & \multicolumn{2}{|c|}{ modal Q } \\
\hline 100 & 104.091 & 101.298 \\
\hline 50 & 53.437 & 51.6233 \\
\hline 20 & \multicolumn{2}{|c|}{35.8147} \\
\hline 10 & \multicolumn{2}{|c|}{86.6343} \\
\hline
\end{tabular}

Table 8: Modal $\mathrm{Q}$ at different values of $\mathrm{Q}_{2}$

Note that as $\mathrm{Q}_{2}$ decreases, the ratio of modal $\mathrm{Q}$ to $\mathrm{Q}_{2}$ increases. 


\section{Literature Cited}

Barbalat, O. (1994). "Industrial and Medical Applications of Accelerators." Frontiers of Accelerator Technology. World Scientific, Singapore. pp. 731-742.

Decking, W. (2001). The TESLA Linear Collider. International Journal of Modern Physics A, vol. 16, Suppl. 1C. World Scientific Publishing Company. 1190-1192.

Henke, H. (1994). "Introduction to High Energy Linear Accelerators for Low Emittance Beams." Frontiers of Accelerator Technology. World Scientific, Singapore. pp. 3-100.

Jones, R.M. (2001). Damping Transverse Wakefields in High Energy Electron Linacs for the JLC/NLC Project. KEK, 2001 Lecture Series, January-March 2001, Unpublished.

Jones, R.M., Baboi, N., \& Kroll, N.M. (2002, June). Multi-Band Dipole and Multiple Wakefields in NLC Traveling Wave Accelerators Using a Wire Measurement Technique. SLAC-PUB-9245.

Jones, R.M., Kroll, N.M., Higo, T., Li, Z., Miller, R.H., Raubenheimer, T.O., \& Wang, J.W. (2001, June). Dipole Wakefield Suppression in High Phase Advance Detuned Linear Accelerators for the JLC/NLC Designed to Minimise Electrical Breakdown and Cumulative BBU. SLAC-PUB-8887.

Jones, R.M., Kroll, N.M., Higo, T., Miller, R.H., \& Ruth, R.D. (2001, June). Manifold Damping of Transverse Wakefields in High Phase Advance Traveling Wave Structures and Local Damping of Dipole Wakefields in Standing Wave Accelerators. SLAC-PUB-8886.

Jones, R.M., Miller, R.H., Wang, J.W., \& Wilson, P.B. (2002, June). Tuning and Field Sensitivity of Pi-mode Standing Wave Linacs for the NLC. SLAC-PUB-9247.

Miller, R.H., Jones, R.M., Adolphsen, C., Bowden, G., Dolgashev, V., Kroll, N., Li, Z., Loewen, R., Ng, C., Pearson, Raubenheimer, T., Ruth, R., Tantawai, S., \& Wang, J.W. (2001, September). Room Temperature Accelerator Structures for Linear Colliders. SLAC-PUB-8889.

Miyamoto, A. (2002, May). JLC Overview. APPI2002 Workshop 2-13-2002 through 2-16-2002.

Nagle, D.E., Knapp, E.A., \& Knapp, B.C. (1967, November). Coupled Resonator Model for Standing Wave Accelerator Tanks. The Review of Scientific Instruments, vol. 38, No. 11, 15831587.

Phinney, N. (2002, June). Next Linear Collider Design Status. SLAC-PUB-9267.

Wilson, P.B. (1989, January). Introduction to Wakefields and Wake Potentials. SLAC-PUB4547.

Yokoya, K. (1986, August). Cumulative Beam Breakup in Large Scale Linacs. DESY 86/084. 